Revue de droit comparé du travail et de la sécurité sociale

$2 \mid 2020$

La vie personnelle du salarié

\title{
Les activités des organes des Nations Unies chargés des droits de l'homme en 2019
}

\section{Elena Sychenko}

\section{(2) OpenEdition}

1 Journals

Édition électronique

URL : https://journals.openedition.org/rdctss/1133

DOI : $10.4000 /$ rdctss. 1133

ISSN : 2262-9815

Éditeur

Centre de droit comparé du travail et de la sécurité sociale

Édition imprimée

Date de publication : 1 juin 2020

Pagination : 130-133

ISSN : 2117-4350

\section{Référence électronique}

Elena Sychenko, « Les activités des organes des Nations Unies chargés des droits de l'homme en 2019 », Revue de droit comparé du travail et de la sécurité sociale [En ligne], 2 | 2020, mis en ligne le 01 novembre 2021, consulté le 11 novembre 2021. URL : http://journals.openedition.org/rdctss/1133 ; DOI : https://doi.org/10.4000/rdctss. 1133

\section{c) (†)}

Revue de droit comparé du travail et de la sécurité sociale est mise à disposition selon les termes de la Licence Creative Commons Attribution - Pas d'Utilisation Commerciale - Pas de Modification 4.0 International. 


\title{
LES ACTIVITÉS DES ORGANES DES NATIONS UNIES CHARGÉS DES DROITS DE L'HOMME EN 2019
}

\begin{abstract}
Traditionnellement, cette rubrique était consacrée aux activités des Comités des Nations Unies créés en vertu des Pactes des droits de l'homme à savoir le Pacte international relatif aux droits civils et politiques et son homologue le Pacte international relatif aux droits économiques, sociaux et culturels. La présente étude s'éloigne de cette tradition et s'intéressera d'abord au cadre de la Convention sur l'élimination de toutes les formes de discrimination à l'égard des femmes (I), puis aux activités pertinentes des organes des Nations Unies en matière de droit du travail dans le cadre des pactes susmentionnés (II).
\end{abstract}

\section{I - LE COMITÉ SUR L'ÉLIMINATION DE TOUTES LES FORMES DE DISCRIMINATION À L'ÉGARD DES FEMMES}

Une affaire très importante a été examinée en $2019^{1}$. La requérante faisait valoir que la Moldavie avait violé ses droits au titre des articles 3 et 11 (2) (c) de la Convention, en ne prenant pas en compte, pour le calcul de sa pension d'assurance sociale, la période de 13 ans durant laquelle elle avait assuré des soins permanents à sa fille handicapée ${ }^{2}$. Elle soulignait le rôle traditionnellement attribué aux femmes dans la société moldave comme principales dispensatrices de soins à leurs enfants handicapés, et affirmait que l'État aurait dû garantir aux femmes la possibilité de combiner leurs obligations en matière de soins aux enfants avec leurs responsabilités professionnelles.

Le Comité a constaté la violation de ses droits et a déclaré que l'exclusion de la période de soins constituait une discrimination indirecte à l'égard des femmes, qui étaient les principales personnes s'occupant de leurs enfants handicapés qui ne bénéficiaient d'aucun service social d'appui leur permettant de combiner les soins aux enfants et le travail. Ces femmes se trouvaient donc privées de la possibilité de cotiser à la caisse d'assurance sociale. Dans ses conclusions, le Comité a demandé à la Moldavie de recalculer la pension d'assurance sociale de la requérante, en tenant compte des 13 années de soins, et de lui accorder une indemnisation au titre des violations subies durant la période où elle a été privée de son droit à la pension d'assurance sociale. Par ailleurs, il a instamment demandé à l'État d'assurer un recours aux autres femmes qui se sont occupées de leurs enfants gravement handicapés pendant la période comprise entre le $1^{\mathrm{er}}$ janvier 1999 et le 31 décembre 2016 lorsque, en vertu de la législation en vigueur, ces périodes n'étaient pas calculées.

1 CEDAW, Natalia Ciobanu v. Republic of Moldova, CEDAW/C/74/D/104/2016, 4 novembre 2019.

2 Prévu par les dispositions de la loi sur les pensions publiques d'assurance sociale, adoptée le 1er janvier 1999. 


\section{ONU - LES DROITS DE L'HOMME EN 2019}

Il s'agit d'une décision remarquable dans la mesure où elle offre une possibilité de recours à des milliers de femmes dont le droit à une pension décente a été violé par la législation du pays en cause en matière de sécurité sociale. Cette affaire peut également être considérée comme un exemple de discrimination par association, peut-être le premier cas de ce type lié à la sécurité sociale.

\section{II - LE COMITÉ DES DROITS ÉCONOMIQUES, SOCIAUX ET CULTURELS (CDESC)}

En 2019, le CDESC a tenu 2 sessions et a examiné les rapports de 11 États $^{3}$ et les communications individuelles de 3 pays différents ${ }^{4}$. Aucune des communications individuelles relatives au droit du travail n'a été jugée recevable. En ce qui concerne les questions examinées dans les observations finales des rapports des pays, deux points d'intérêt méritent une attention particulière.

\section{A - ENTREPRISES ET DROITS DE L'HOMME}

L'examen des observations finales montre que le CDESC pousse les États développés à mettre en œuvre, dans leur législation et leur pratique nationales, les Principes directeurs de I'ONU sur les entreprises et les droits de l'homme (2011) ainsi que les objectifs de développement durable portés par l'ONU. II s'agit d'une étape remarquable dans la promotion de ces initiatives purement volontaires, permettant de convaincre les États de prendre au sérieux les violations des droits de l'homme commises par les multinationales à l'étranger. La question de la diligence raisonnable est devenue, assez récemment, un sujet de droit national ${ }^{5}$ et l'inclusion de la recommandation visant à développer ces systèmes dans les observations finales du CDESC permettraient de faire de ces lois une règle, plutôt qu'une exception.

Ainsi, en ce qui concerne le Danemark, le Comité s'est inquiété du fait qu'une obligation de diligence raisonnable en matière de droits de l'homme ne soit pas imposée aux entreprises domiciliées dans sa juridiction, et il a donc recommandé à l'État d'adopter un cadre qui :

- oblige les entreprises à faire preuve de la diligence requise en matière de droits de I'homme, dans leurs opérations et dans leurs relations commerciales, dans leur pays et à l'étranger ;

- tienne les entreprises responsables des violations des droits économiques, sociaux et culturels ;

- permette aux victimes d'exercer des recours par le biais de mécanismes judiciaires et non judiciaires dans l'État partie.

3 Session 66: Danemark, Équateur, Israël, Sénégal, Slovaquie; session 65 : Suisse, Bulgarie, Cameroun, Estonie, Kazakhstan, Maurice. Voir : https://tbinternet.ohchr.org/layouts/15/TreatyBodyExternal/ SessionsList.aspx? Treaty $=$ CESCR

4 Espagne, Italie, Luxembourg.

5 Voir : la loi britannique sur l'esclavage moderne adoptée en 2015; la lettre d'intention française n²017-399 relative au devoir de vigilance des sociétés mères et des entreprises donneuses d'ordre adoptée en 2017; la loi néerlandaise sur la diligence raisonnable en matière de travail des enfants adoptée en 2019; la directive européenne sur l'information non financière 2014/95/UE. 
La Suisse a été également critiquée pour n'avoir introduit la diligence raisonnable que sur une base volontaire. Aussi, lui a-t-il été recommandé de prendre des mesures quasiment identiques à celles du Danemark (évoquées ci-dessus).

Parallèlement, s'agissant des pays en développement, le CDESC s'est inquiété du manque de données complètes permettant de garantir que les entreprises appliquent la diligence raisonnable en matière de droits de l'homme (notamment au Kazakhstan). Par exemple, il a été recommandé au Cameroun d'évaluer l'impact sur les droits de l'homme des projets de développement économique, y compris ceux mis en œuvre par des acteurs privés. Fondamentalement, cette approche vise à garantir la participation des États hôtes et bénéficiaires des investissements de la multinationale aux processus de diligence raisonnable et de conformité de leurs activités avec les normes internationales en matière de droits de l'homme. Ces normes, grâce au travail de promotion des différents organismes de défense des droits de l'homme (références croisées des instruments internationaux et des conclusions des organismes concernés, par exemple), constituent désormais une sorte de bloc de normes unifié, même si elles sont portées par différents instruments internationaux.

Cette unité est clairement visible dans les observations finales du CDESC, qui ont tendance à se référer à d'autres conventions des Nations Unies, aux conventions de l'OIT. II convient également de noter que le Pacte ne contient aucune disposition sur la diligence raisonnable, par conséquent, tous les commentaires du CDESC relatifs à ce sujet sont une sorte d'interprétation " évolutive » conforme au développement des initiatives en matière de droits de l'homme au sein des Nations Unies.

\section{B - LES DROITS DES SYNDICATS}

Les droits des syndicats, la sécurité des syndicalistes, ainsi que la réalisation du droit de grève constituaient un autre point commun des observations finales de 2019.

Au Sénégal, par exemple, le CDESC a souligné que le droit du ministère de l'Intérieur de délivrer un reçu reconnaissant l'existence d'un syndicat, après s'être assuré que ses membres sont de bonne moralité et ne sont pas en conflit avec la loi, n'est pas conforme à la Convention n ${ }^{\circ} 87$ de l'OIT. Au Cameroun, la loi antiterroriste interdit les actes susceptibles de perturber le fonctionnement normal des services publics ou des services essentiels (la peine de mort en est la sanction) et, par conséquent, le Comité estime qu'elle impose une restriction importante aux activités syndicales. Le CDESC a exhorté ces deux pays à modifier la législation conformément à la Convention n87 de l'OIT.

Les pays développés ont été critiqués pour ne pas avoir autorisé les fonctionnaires à exercer leur droit de grève ou à prendre part à d'autres actions collectives (Estonie) ${ }^{6}$. Concernant la Slovaquie, le CDESC a noté avec inquiétude que les travailleurs licenciés pour leur participation à des activités syndicales n'avaient pas droit à la réintégration. II est intéressant de noter que cette question n'a jamais été portée à l'attention du Comité de la liberté syndicale de l'OIT.

6 Le Comité de l'OIT sur la liberté d'association a examiné la revendication d'une telle interdiction, et a recommandé à l'Estonie de veiller à ce que les fonctionnaires, qui n'exercent pas l'autorité au nom de l'État, jouissent du droit de grève, en 2007. Il semble que rien n'ait changé depuis lors. Voir OIT CFA, Rapport définitif - Rapport n³50, juin 2008, Affaire n²543 (Estonie) - Date de la plainte : 31-Jan-07. 


\section{ONU - LES DROITS DE L'HOMME EN 2019}

L'examen des observations finales montre que le CDESC a révélé des pratiques et des normes antisyndicales dans la majorité des pays en développement dont il a examiné les rapports en 2019. II semblerait que ces restrictions persistent dans la mesure où elles constituent un avantage concurrentiel de certains Etats permettant d'attirer des capitaux étrangers.

\section{C - Le Comité des dROITS DE L'HOMME}

En 2019, le Comité des droits de l'homme (CDH) a tenu 3 sessions (125-127) examinant au total 13 rapports des Etats et un certain nombre de communications individuelles. Aucune de ces communications n'avait de rapport avec le droit du travail.

Cependant, dans les observations finales, il existe traditionnellement un bloc très directement lié avec le droit du travail : les mesures prises par les États pour lutter contre la traite des êtres humains. En passant en revue les activités du CDH depuis un certain nombre d'années, on peut en conclure que cette question a toujours constitué un problème pour tous les États, en particulier pour les pays en développement. En 2019, le CDH a de nouveau exhorté un certain nombre d'États à intensifier leurs efforts pour prévenir, combattre et punir la traite des personnes. Il a constaté qu'au Mexique, les migrants sont particulièrement vulnérables et ne bénéficient pas d'une protection et d'une assistance efficaces de la part de l'État. Le Sénégal a pour sa part été invité à donner aux organisations de la société civile la possibilité d'engager des actions civiles devant les tribunaux, et à garantir qu'une assistance juridique soit fournie à toutes les victimes de la traite. En ce qui concerne l'Angola ${ }^{7}$, le CDH a souligné la nécessité de prendre des mesures pour éliminer le travail forcé et le travail des enfants - en particulier dans le secteur minier - notamment en augmentant la capacité des inspecteurs du travail et en allouant des ressources appropriées à l'administration du travail. Le Vietnam ${ }^{8}$, qui a été rétrogradé au niveau 2 de la liste de surveillance dans le rapport américain sur la traite des êtres humains en 2019, a été critiqué pour l'accès limité des victimes aux services sociaux.

Le Paraguay a été invité à combattre la pratique du «criadazgo »- une forme d'esclavage moderne où les enfants sont exploités pour le travail domestique. II s'agit d'un " travail invisible ", comme l'a noté un jour I'UNICEF'. Le HRC a recommandé à l'État d'adopter des instruments réglementaires et des politiques visant à l'éliminer, notamment le soutien à la famille d'origine, l'élaboration de campagnes de sensibilisation et de programmes d'éducation et de formation professionnelle pour les enfants et les adolescents issus de familles vulnérables dans tout le pays.

Pour conclure, il faut signaler qu'au cours de toutes les sessions, un certain nombre de pays n'ont pas fourni les rapports aux Comités concernés en dépit de leurs obligations internationales. De toute évidence, la communauté internationale devrait trouver le moyen de renforcer le respect des États vis-à-vis de leurs obligations en matière de rapports, peut-être en utilisant la pression du FMI ou de la Banque Mondiale.

7 Tier 2 Watch List, 2019, Trafficking in Persons Report : https://www.state.gov/wp-content/ uploads/2019/06/2019-Trafficking-in-Persons-Report.pdf

8 Ibid.

9 Niñez y adolescencia trabajadora en Paraguay, UNICEF, 2004 : https://www.unicef.org/paraguay/ media/2046/ file/ninez-trabajadora.pdf 